\title{
BROOM: An International Magazine of the Arts (1921-1924) : une revue d'avant-garde américaine
}

Broom: An International Magazine of the Arts (1921-24), an American avant-garde magazine

\section{Ambre Gauthier}

\section{(2) OpenEdition} Journals

Édition électronique

URL : http://journals.openedition.org/cel/504

DOI : $10.4000 / \mathrm{cel} .504$

ISSN : 2262-208X

Éditeur

École du Louvre

Édition imprimée

Date de publication : 1 octobre 2013

Référence électronique

Ambre Gauthier, «BROOM: An International Magazine of the Arts (1921-1924) : une revue d'avant-garde américaine », Les Cahiers de l'École du Louvre [En ligne], 3 | 2013, mis en ligne le 01 octobre 2013, consulté le 19 avril 2019. URL : http://journals.openedition.org/cel/504; DOI : 10.4000/cel.504

Les Cahiers de l'École du Louvre sont mis à disposition selon les termes de la licence Creative Commons Attribution - Pas d'Utilisation Commerciale - Pas de Modification 4.0 International. 


\title{
Cahiers de l'École du Louvre recherches en histoire de l'art, histoire des civilisations archéologie, anthropologie et muséologie \\ Numéro 3. Octobre 2013
}

\author{
BROOM: An International Magazine of the Arts \\ (1921-1924) : une revue d'avant-garde américaine \\ Ambre Gauthier
}

Article disponible en ligne à l'adresse :

http://www.ecoledulouvre/revue/numero3octobre2013/Gauthier.pdf

Pour citer cet article :

Coline Ambre Gauthier, «BROOM : An International Magazine of the Arts (19211924) : une revue d'avant-garde américaine », Cahiers de l'École du Louvre, Recherches en histoire de l'art, histoire des civilisations, archéologie, anthropologie et muséologie [en ligne] n 3, octobre 2013, p. 24 à 35.

(C) École du Louvre

Cet article est mis à disposition selon les termes de la Licence Creative Commons Attribution - Pas d'utilisation commerciale - Pas de modification 3.0 non transposé. 


\section{Cahiers de l'École du Louvre recherches en histoire de l'art, histoire des civilisations archéologie, anthropologie et muséologie \\ Numéro 3. Octobre 2013}

\section{Sommaire}

\section{Éditorial}

Équipe de recherche

\section{Dossier: les revues}

Le « GR05 ». Approche de quelques revues du XX $\mathrm{XX}^{\mathrm{e}}$ siècle Introduction d'Hélène Klein

La présentation des objets africains dans DOCUMENTS (1929-1930), magazine illustré

Coline Bidault

Le conflit renaissant de la figure et de l'abstraction dans Labyrinthe, journal mensuel des Lettres et des Arts (octobre 1944-décembre 1946)

Blandine Delhaye

BROOM: An International Magazine of the Arts (1921-1924):

une revue d'avant-garde américaine

Ambre Gauthier

L'image de la guerre dans L'Élan (1915-1916), un refoulement apparent Hadrien Viraben

\section{Articles}

" John Constable et le statut de l'esquisse »

Conférence du 9 avril 2013 dans le cadre du séminaire doctoral

John Murdoch

«Adieu veau, vache, cochon, couvée... » La boucherie à l'Ancien Empire : croisement des données iconographiques, textuelles et archéologiques

Fanny Hamonic

L’entreprise patrimoniale de Louis-Joseph GUYOT (1836-1924)

à Dourdan, entre érudition et médiation

Léda Martines

p. $63-72$

\section{Actualité de la recherche}

Reconnaissance et médiation d'un patrimoine : vecteurs d'une identité territoriale? Journées d'étude de troisième cycle, 14 et 15 juin 2012

Noémie Couillard, Nicolas Navarro, Maylis Nouvellon p. $73-81$

Les « Ymagiers » à l'École du Louvre

Cycle de conférences organisées par l'IRHT 


\section{Ambre Gauthier}

\section{La naissance d'une little review entre New York et Rome}

La revue américaine Broom ${ }^{1}$ paraît de novembre 1921 à janvier 1924. Publiée à l'initiative d'un héritier de la famille Guggenheim, Harold A. Loeb (1871-1974) et par ses collaborateurs, elle compte vingt-et-un numéros mensuels (fig. 1).
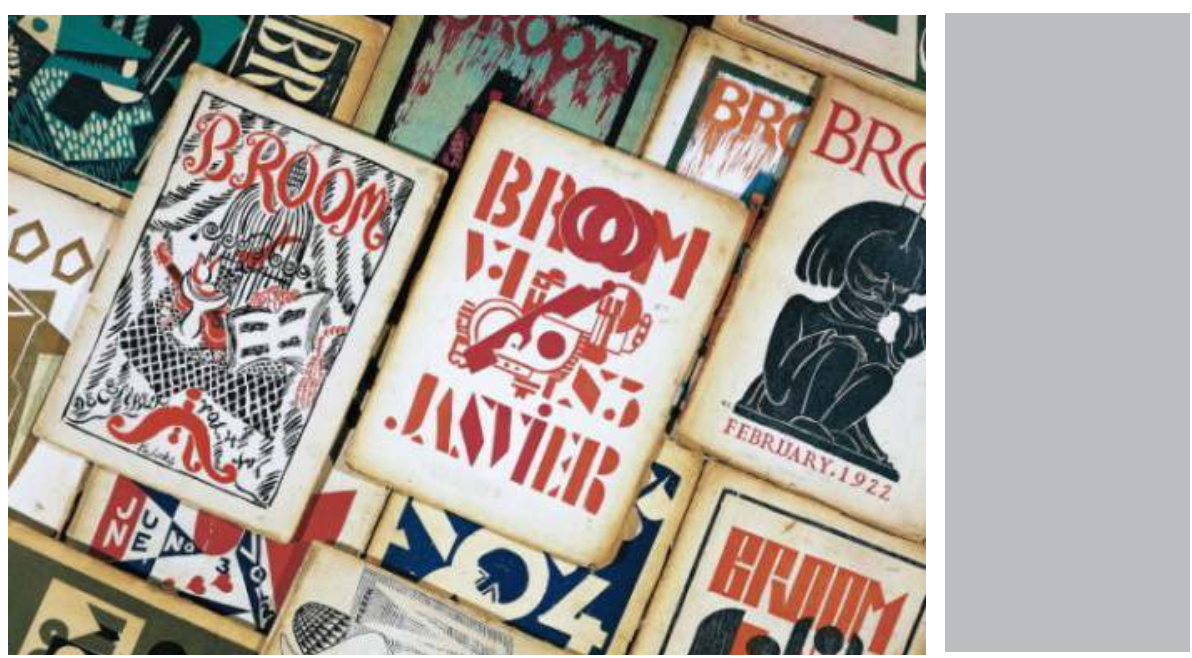

La correspondance, inédite ${ }^{2}$, riche de plusieurs milliers de lettres échangées entre H. Loeb, les éditeurs de Broom et la famille Guggenheim, a permis de retracer l'aventure éditoriale de la revue de sa genèse à son arrêt brutal. Cette source primaire de la recherche a été confrontée avec les écrits autobiographiques des éditeurs H. Loeb, The Way it Was ${ }^{3}$ (1959), Matthew Josephson, Life among the Surrealists ${ }^{4}$ (1962) et Alfred Kreymborg, Troubadour ${ }^{5}$ (1925) pour tenter de reconstruire de manière précise l'itinéraire mouvementé de la revue publiée entre Rome, Berlin et New York. S'inscrivant pleinement dans le phénomène de publication des petites revues littéraires américaines, les little reviens, sa publication est « délocalisée » dès le premier numéro, à la fois par souci d'économie et en raison de l'intention ferme de ses éditeurs de se rapprocher de l'Europe, du Vieux Continent, source d'inspiration non démentie des Américains, de ceux que Gertrude Stein nomme la Lost Generation, la génération perdue. La revue est initialement publiée à Rome de novembre 1921 à octobre 1922, puis à Berlin de décembre 1922 à mars 1923, et enfin à New York d'août 1923 au dernier numéro paru en janvier 1924'. D'abord intitulée Broom: An International Magazine of the Arts published by Americans in Italy lors de sa publication à Rome (jusqu'au volume II, $\mathrm{n}^{\circ}$ 1, du mois d'avril 1922, dernier à être édité en Italie), elle opte pour un sous-titre plus synthétique en mai 1922, après la démission de

\footnotetext{
${ }^{1}$ Broom: An International Magazine of the Arts published by Americans in Italy, Rome, New York, Berlin, Harold A. Loeb, Alfred Kreymborg, 1921-1924. Il existe un reprint de la revue, édité en 1967 par Kraus. L'étude de cette revue a été rendue possible par la mise à disposition de la correspondance de l'éditeur H. Loeb par la Princeton Library.

2 La correspondance d'Harold Loeb, intitulée Broom Correspondance of Harold Loeb, est conservée à la Princeton Library sous la cote C0110. Pour des raisons de propriété intellectuelle, aucune lettre de cette correspondance ne sera reproduite dans cet article.

${ }^{3}$ Harold A. Loeb, The Way it Was, New York, Criterion Books, 1959.

${ }^{4}$ Matthew Josephson, Life among the Surrealists, New York, Holt, Rinehard \& Winston, 1962.

${ }^{5}$ Alfred Kreymborg, Troubadour, New York, Boni \& Liveright, 1925.

${ }^{6}$ On dénombre ainsi onze numéros édités à Rome (du volume I, $\mathrm{n}^{\circ} 1$ au III, $\mathrm{n}^{\circ} 3$ ), cinq numéros publiés à Berlin (volume III, n० $4-\mathrm{IV}, \mathrm{n}^{\circ} 4$ ) et enfin, cinq autres publiés à New York (du volume $\mathrm{V}, \mathrm{n}^{\circ} 1$ au $\left.\mathrm{VI}, \mathrm{n}^{\circ} 1\right)$.
} 
l'éditeur associé Alfred Kreymborg, devenant Broom: An International Magazines of the Arts published by Harold Loeb (même si Loeb ne reste éditeur unique que pour les deux premiers numéros du volume II). Son titre énigmatique, Broom, est une référence revendiquée à un passage de l'ouvrage Moby Dick d'Herman Melville (1851) : « Qu'en est-il, si certains vieux bellâtres de capitaines m’ordonnent de prendre un balai et de balayer le pont ? Qu'est-ce que cette humiliation comparée, pesée, je veux dire, dans les balances du Nouveau Testament? Pensez-vous que l'archange Gabriel me tienne en moindre estime, parce que promptement et respectueusement je me suis empressé d'obéir à ce vieux capitaine en cette occasion particulière ? Qui n'est pas un esclave ? ${ }^{7} \gg$ Le mot broom, qui signifie littéralement «balai », est le titre retenu par Loeb et Kreymborg dès le début de la création de la revue. À la recherche d'un mot fort, d'un nom monosyllabique percutant, ils s'entendent sur broom en parcourant les pages du roman de Melville. Cet ouvrage, véritable manifeste du roman classique américain, fait écho à la qualité littéraire attendue dans la revue. La symbolique dynamique du balai sied particulièrement bien à la jeune little review, qui entend balayer les conventions pour dépoussiérer l'art américain de ses influences passéistes et s'orienter vers un art nouveau, tournant ainsi le dos au puritanisme ambiant. Le choix de cette image rustique, associée au travail des classes ouvrières et aux milieux modestes, est révélateur de la condition sociale de Loeb à cette époque, qui mène, selon ses dires, une vie de « bohémien miséreux ». La citation de Moby Dick est reproduite dès le premier numéro en quatrième de couverture, associée à un singulier cachet dessiné par l'écrivain Gordon Craig (fig. 2) : un petit personnage dansant avec un balai à la main, qui accentue l'idée de renouveau émise par le titre de la revue. Cette volonté de rupture avec les conventions et traditions (littéraires, artistiques, sociologiques) se retrouve également dans le choix des couvertures de la revue. La couverture du volume I, numéro 4, février 1922, (fig. 3) également réalisée par Gordon Craig, demeure l'exemple le plus pertinent de cet archaïsme volontaire. La gravure montre un personnage démoniaque, défiant le lecteur non averti en lui faisant un pied de nez. L'audace graphique, alliée à l'archaïsme de la gravure sur bois et à la provocation du geste, ne font qu'amplifier « le profond désir de défier la norme ${ }^{8} »$. Titre et graphisme concourent donc à poser les fondements d'un programme littéraire et artistique bien défini, qui prend toute sa dimension dans l'élaboration de la ligne éditoriale.
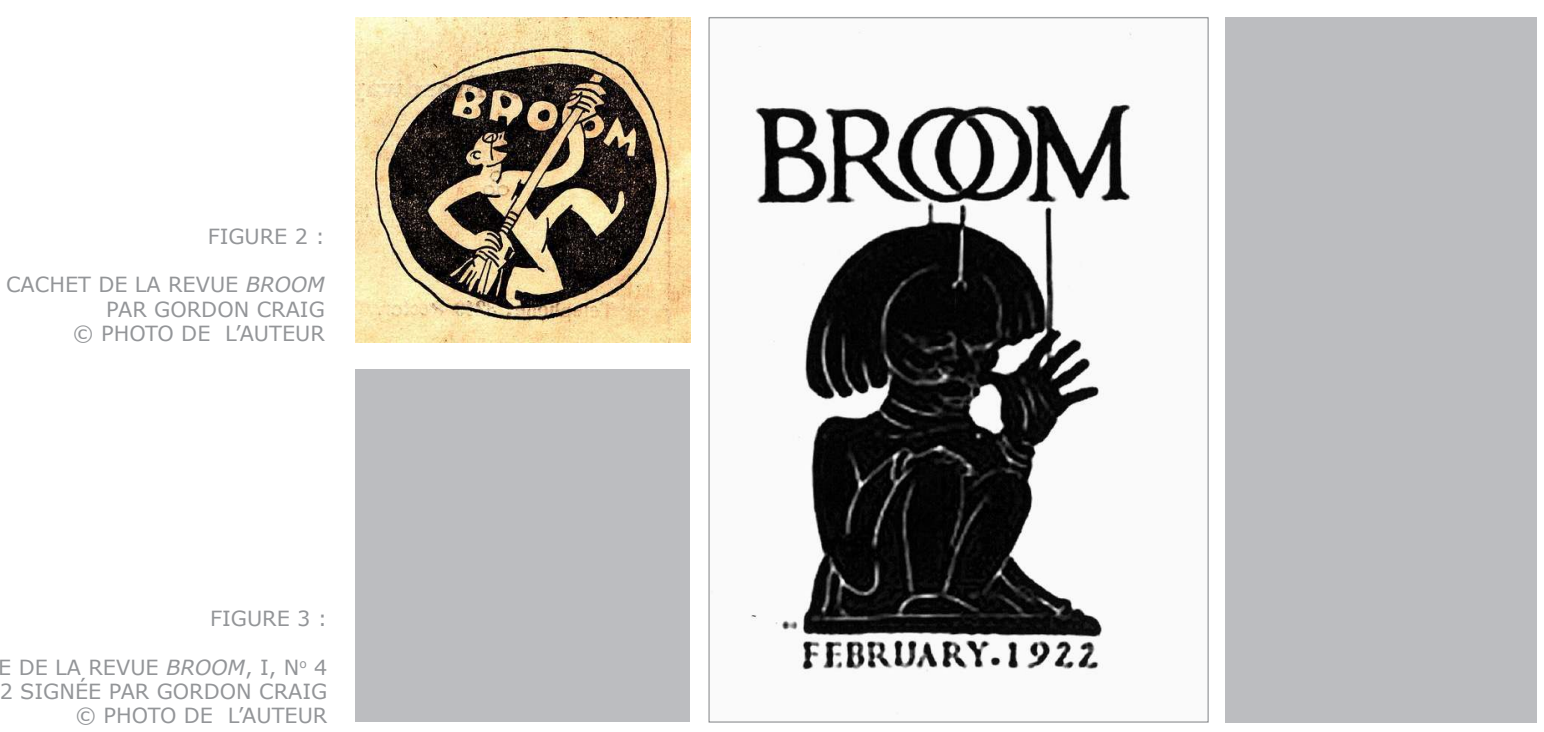

7 Herman Melville, Moby Dick, Penguin Classics, New York, 1994, p. 20 : "What of it, if some old hunks of a sea-captain orders me to get a broom and sweep down the decks? What does that indignity amount to, weighed, I mean, in the scales of the New Testament? Do you think the archangel Gabriel thinks anything the less of me, because I promptly and respectfully obey that old hunk in that particular instance? Who ain't a slave?"

8 Paul Finkelman et Cary D. Wintz, Encyclopedia of the Harlem Renaissance, Volume 1, Routledge, Cary D. Wintz and Paul Finkelman, 2004, pp. 187-188 : « Le dessin de couverture de Gordon Craig pour le quatrième numéro, représentant un personnage faisant un pied de nez, donnait à voir une volonté de défier la norme toujours plus forte »; "Gordon Craig's cover drawing for the fourth issue, of a figure thumbing its nose, implies an even stronger sense of defying the norme". 
La revue nait dans l'effervescence provoquée conjointement par l'essor des little magazines ${ }^{9}$ aux États-Unis mais aussi par les débats issus des soirées littéraires organisées par la librairie The Sunwise Turn à New York. Riche de ses contributeurs internationaux et de ses éditeurs successifs, elle ne compte pas moins de cent-quatrevingt-dix-sept collaborateurs et dix éditeurs. Soucieux de produire une revue littéraire et artistique moderniste de qualité, Loeb s'associe au poète et éditeur Alfred Kreymborg (1883-1966) dès le mois de février 1921. Ce dernier, fort de plusieurs expériences éditoriales, a déjà édité deux revues modernistes : The Glebe $^{10}$ (1913-1914) avec Man Ray à New York et Ridgefield, et Others : A Magazine of the New Verse $e^{11}$ (1915) à Ridgefield. Il est l'éditeur associé des premiers temps de Broom, celui qui officie avec Loeb à Rome jusqu'en décembre 1921. Il est l'homme par qui les rencontres avec écrivains et artistes se font, son réseau social et sa réputation dans les milieux littéraires permettant des associations fructueuses. Leurs divergences d'opinions sur le modernisme et le nationalisme américain mettent fin à cette collaboration. Loeb s'ouvre à l'Europe et à ses avant-gardes après son premier séjour en France en 1921, tandis que Kreymborg reste concentré sur la vocation première de la revue, qui est de promouvoir la littérature américaine et ses jeunes auteurs. C'est ainsi que « des tensions ont effectué leur entrée dans les bureaux. Harold se tournait de plus en plus vers l'Europe et les auteurs à la réputation faite et Krimmie (Kreymborg) vers l'Amérique et le futur ${ }^{12}$ ». À Rome, les problèmes d'impression et d'acheminement des exemplaires de la revue vers les États-Unis se multiplient. Dans deux lettres datées des 2 et 3 décembre $1921^{13}$, Kreymborg explique l'infortune dont Broom a été victime : après un retard considérable d'impression, les exemplaires du numéro 2 ont été oubliés à l'imprimerie par le secrétaire d'édition, et n'ont jamais pu être expédiés jusqu’à New York. Loeb, à Paris en 1921, noue des relations étroites avec Kathleen Eaton Cannell (1891-1974, dite Kitty Cannell). Muse du Tout-Paris, danseuse et journaliste, ses connaissances mondaines et son goût littéraire la conduisent à occuper des fonctions éditoriales dans la revue du printemps de 1921 à janvier 1924. Loeb lui réserve une place de choix dans son autobiographie ${ }^{14}$ sous le pseudonyme de Lily Lubow, n'hésitant pas à dresser un portrait féroce de celle qui a été sa compagne, dont les crises de jalousie et les prises de position drastiques influencent l'édition déjà mouvementée de la revue. Pour Kitty Cannell, l'art devrait être désacralisé, pour devenir une source «d'amusement» car ce « serait une erreur de considérer l'art comme sacro-saint, même si le meilleur de l'art est capable de produire une réaction émotionnelle beaucoup plus intense qu'aucune autre expérience humaine ${ }^{15} »$. Les Américains " étaient trop sérieux à ce sujet », tandis qu'en France, « cuisiner était considéré comme étant aussi important que la peinture dans certains cercles ${ }^{16} »$. Cannell travaille anonymement aux côtés de Loeb pour Broom de 1921 à 1924, jouant le rôle de conseillère artistique et d'assistante éditoriale non-officielle. Car avant

\footnotetext{
${ }^{9}$ Les little magazines ou little reviews sont des périodiques à visée non commerciale et à tirage limité, généralement utilisés comme moyens de diffusion de la littérature et de l'art expérimental, véhiculant le plus souvent des théories sociales ou politiques révolutionnaires, non conventionnelles. Leur essor commence dans les années 1910, résultant de la symbiose entre une profusion d'idées et de technologies nouvelles. Il participe et nourrit les revendications du mouvement moderniste américain.

${ }_{10}$ The Glebe est une revue littéraire publiée entre septembre 1913 et novembre 1914, comportant dix numéros. Sous I'autorité d'Alfred Kreymborg et de Man Ray, sa ligne éditoriale se concentre sur la littérature et la poésie moderniste contemporaine, la publication du manifeste imagiste « Des Imagistes : An Anthology » d’Ezra Pound paru au volume I, n 5 (février 1914) I'ayant rendue célèbre.

${ }^{11}$ Others: A Magazine of the New Verse est un magazine poétique publié par Alfred Kreymborg de juillet 1915 à juillet 1919, grâce au financement du mécène Walter Conrad Arensberg. Souhaitant promouvoir la nouvelle poésie moderniste américaine, ses contributeurs réguliers sont William Carlos Williams, Wallace Stevens, Marianne Moore, Mina Loy, Conrad Aiken, Carl Sandburg, T. S. Eliot, Amy Lowell, Man Ray, Lola Ridge, auteurs qui collaboreront plus tard à Broom. 12 A. Kreymborg, op.cit. note 5, p. 301 : "But friction has effected an entrance into the editorial offices. Harold inclined more and more toward Europe and established reputations and Krimmie toward America and the future".

13 Lettres d'Alfred Kreymborg à Harold A. Loeb, 2 et 3 décembre 1921, Broom Correspondence of Harold Loeb, Princeton Library.

${ }^{14} \mathrm{H}$. A. Loeb, op. cit. note 3.

${ }^{15}$ Id., Ibid., p. 65 : "It was a mistake to treat art as sacrosanct, even thought as its best it was capable of evoking a response far more intense than that of any other human experience".

${ }^{16}$ Id., Ibid., pp. 64-65: "Art, she said, should first of all 'amuse' [...]. We were too serious about it in America" ; "Cooking was considered as important as painting in certain French circle".
} 
d'arriver à Paris, elle a été critique à New York pour la revue The Christian Science Monitor. Brièvement mariée au poète Skipwith Cannell, elle divorce en 1921 et va dès lors partager librement la vie d'Harold Loeb jusqu'en 1924. Sa position influente dans les milieux artistiques parisiens permet de doter Broom de contributions variées (Lipchitz, Brancusi, Picasso), Kitty Cannell servant d'intermédiaire entre éditeurs et artistes, usant de son charme et de sa culture pour convaincre les deux parties de s'entendre. Son goût personnel oriente considérablement la revue vers les avant-gardes européennes, avant-gardes méconnues de Loeb avant ses séjours à Paris. Altruiste et passionnée, elle est la femme de l'ombre de la revue, son nom n'étant mentionné dans aucun sommaire malgré l'importance de son travail. Cependant, de l'autre côté de l'Atlantique, un éditeur américain est engagé pour réunir les contributions américaines, Nathaniel Shaw. Il officie du 12 juillet 1921 au mois de février 1922 avant d'être remercié par Loeb, celui-ci le jugeant peu réactif et peu impliqué. Il est rapidement remplacé par la poétesse Lola Ridge (1873-1941), qui prend ses fonctions le $1^{\text {er }}$ mars 1922. Engagée politiquement et servant la cause féministe, elle publie ses poèmes dans la majeure partie des little reviews américaines (Poetry, The New Republic, The Saturday Review of Literature). Son premier recueil The Ghetto and Other Poems parait en 1918, salué unanimement par la critique. Il en va de même pour Sun-up and Other Poems en 1920, et The Red Flag en 1927, ce qui la positionne en figure incontournable de la poésie moderniste féministe. Simultanément, l'homme de théâtre Edward Storer (1880-1944) est embauché en tant qu'éditeur associé. Celui-ci, détenteur des droits de l'œuvre théâtrale de Luigi Pirandello (1867-1934), permet à la revue de publier une traduction de Six Characters in Search of an Author ${ }^{17}$ même si Loeb refuse d'en acheter les droits. Cette association dure jusqu'à l'automne 1922, où Storer est remplacé par Matthew Josephson en même temps que la revue déménage de Rome à Berlin. Après des études à l'université de Columbia, Josephson avait été correspondant et journaliste pour de nombreux magazines américains en Europe. En 1922, avant de rejoindre la rédaction de Broom, il collabore activement à la revue Secession, éditée par son ami Gorham B. Munson. Maitrisant le français et introduit dans la plupart des cercles littéraires parisiens, il côtoie Louis Aragon, Philippe Soupault et Benjamin Péret, qui demeureront des amis fidèles pendant de longues années. Josephson, dans ses mémoires, évalue son apport considérable au contenu de la revue : "Je préconisais que Broom devrait essayer de se distinguer des autres publications en présentant des contributions littéraires aussi audacieuses que possible et aussi modernes que l'art de Picasso et de l'École de Paris. Le mouvement Dada était en train de s'éteindre officiellement, mais ses membres français, comme Aragon et Éluard, semblaient au commencement de grandes carrières littéraires, et particulièrement appropriés pour être publiés dans Broom. Je proposais également des traductions d'auteurs plus anciens, comme Apollinaire et Lautréamont ${ }^{18} »$. Ces amitiés lui permettent de faire bénéficier la revue Broom de nombreuses contributions, dont celle de la poétesse et collectionneuse Gertrude Stein. Dans une lettre du 29 novembre 1922, de H. Loeb à Gertrude Stein ${ }^{19}$, l'on apprend que l'éditeur, sur les recommandations de Josephson, a lu les poèmes de la poétesse et qu'il les a tant aimés, qu'il souhaite les publier très prochainement dans Broom. La publication de poèmes de Gertrude Stein au volume IV, numéro 2 (janvier 1923), provoque une querelle décisive

17 Six Characters in Search of an Author est la traduction de la pièce Sei Personaggi in Cerca d'Autore (1921) de Luigi Pirandello (1867-1934), adaptée par Edward Storer lui-même pour la version anglaise. Cette célèbre pièce, mise en abîme satyrique du monde théâtral, est publiée dans les volumes II, no 3 (juin 1922, p. 186), II, no 4 (juillet 1922, p. 321), et enfin, III, n 1 (août 1922 , p. 44). Storer, détenteur des droits américains de l'œuvre de Pirandello, proposera à Loeb de les lui vendre pour 1000 \$ en 1922. Loeb fera le choix de ne pas les acquérir, pensant à l'insécurité d'un tel placement. Au regard du succès de Pirandello et de son Prix Nobel de littérature reçu en 1934, le choix de Loeb fait apparaître une erreur de jugement regrettable, ces royalties auraient pu assurer une rentrée d'argent régulière et pérenne à Broom.

$18 \mathrm{M}$. Josephson, op. cit. note 4, pp. 169-170 : "I urged that Broom should try to distinguish itself from other publications by presenting literary material that was challenging as possible and at least as 'modern' as the art of Picasso and the School of Paris. The Dadaist movement was reported to be expiring officially, but its French members, such as Aragon and Eluard, seemed to be at the beginning of great literary careers, and very suitable for inclusion in Broom. I also proposed translations from earlier authors, such as Apollinaire and Lautréamont".

19 Lettre d'Harold Loeb à Gertrude Stein, 29 novembre 1922, Gertrude Stein Papers, Yale University Library Special Collections. 
entre Loeb et l'éditrice Ridge : elle démissionne à la suite de cette publication qui va à l'encontre de ses convictions littéraires, pensant que si «Gertrude Stein a une lueur occasionnelle, c'est la plupart du temps du blablabla ${ }^{20} »$. À Berlin, les coûts de production de la revue se font de plus en plus lourds, malgré la dévaluation du mark, très favorable aux américains. Harold Loeb chiffre à l'automne 1922 entre 860 à 960 \$ pour la production d'un numéro, soit 3000 exemplaires. Il estime que les gains possibles peuvent atteindre $1050 \$$ si la totalité des exemplaires sont écoulés, et davantage si les demandes d'abonnement sont nombreuses (pouvant rapporter jusqu'à 100 \$ par mois). Le problème majeur réside dans la vente des 3000 exemplaires, qui ne s'écoulent jamais complètement. Malgré ces difficultés financières, l'artiste hongrois Ladislaw Medgyes rejoint au cours de l'hiver 1923 H. Loeb, Ridge et Josephson, en tant qu'éditeur chargé de développer le pôle artistique de la revue. Rapidement, les relations se détériorent entre Harold Loeb et Lola Ridge, la rigueur et la fermeté des jugements de l'écrivain américaine s'accordant difficilement avec la vocation libertaire de Broom. Leur collaboration s'arrête brutalement en mars 1923, en même temps que celle avec Medgyes. Le déménagement de la revue à New York au printemps 1923 s'accompagne d'une restructuration du personnel : H. Loeb conserve sa position d'éditeur principal, Josephson demeure son éditeur associé et embauche deux éditeurs supplémentaires, Malcolm Cowley (1898-1989) et William Slater Brown (1896-1997) qui travaillent pour la revue jusqu'à son dernier numéro, publié en janvier 1924.

Si la parution de Broom se voulait initialement mensuelle, on observe néanmoins que dès l'année 1922, les difficultés financières et matérielles de la revue ne permettent pas toujours de soutenir ce rythme. Ainsi, il n'y a pas de numéro publié en mars 1922. De même, les difficultés croissantes de financement vont stopper la publication entre le numéro de mars 1923 et d'août $1923^{21}$. Le rythme mensuel de publication est rendu possible par le travail simultané des éditeurs sur plusieurs numéros en même temps : les contributions collectées sont réparties sur deux ou trois numéros pour présenter un ensemble homogène de qualité égale, et lorsqu'un exemplaire peut passer sous presse, un ou deux exemplaires à venir sont déjà en train d'être complétés ou sont quasiment achevés. Ce travail de composition complexe, souvent aléatoire, n'exclut pas les envois de dernière minute à intégrer, ni les retards de publication par manque de contributions de qualité. Ainsi, le 22 novembre 1922, les éditeurs utilisent une deuxième fois la couverture exécutée par Enrico Prampolini pour le premier numéro, celle qui devait être réalisée par un artiste tchèque n’étant pas arrivée dans les temps pour l'impression du second numéro (celui-ci devant paraitre début décembre 1922). De même, le volume III, numéro 3 (octobre 1922) devait contenir des photographies inédites de Paul Strand (1890-1976), pour soutenir la réflexion sur l'industrialisation et le modernisme des États-Unis, sujet récurrent de la revue. Ces photographies, à l'esthétique épurée, se composent de plans fixes cherchant à capter avec objectivité l'essence même du monde industriel, des usines, des chaines de production, des machines, sans avoir recours aux effets de flou et de brouillard tant appréciés par bon nombre de ses contemporains ${ }^{22}$. Cette esthétique de la machine est une des prérogatives de la revue, comme le montre la couverture du numéro 3 (janvier 1922), réalisée par Fernand Léger (fig. 4). La qualité des reproductions des photographies de Paul Strand étant insuffisante, Loeb décide de les remplacer au dernier moment par une série de dessins d'Henri Matisse (1869-1954), représentant essentiellement des portraits de femmes, pour que l'exemplaire puisse être imprimé à temps. La thématique industrielle du numéro devient caduque, les photographies sont différées au numéro suivant,

20 Lettre de Lola Ridge à Harold Loeb, 11 juillet 1922, Broom Correspondence of Harold Loeb, Princeton University Library: "Gertrude Stein has only an occasional gleam - it is mostly blah! blah!".

${ }^{21}$ La publication de la revue devait alors s'arrêter définitivement après la parution du numéro de mars 1923 comme l'atteste une lettre de Lola Ridge adressée à Harold A. Loeb le 7 février 1923, Broom Correspondence of Harold Loeb, Princeton University Library : les oncles de Loeb, qui étudiaient la question du financement possible de la revue, ont décidé de ne plus investir dans cette entreprise, la condamnant à une fin imminente. Les éditeurs ne se résigneront pas et établiront un nouveau plan de financement pour faire renaître la revue à New York dès l'été 1923. 22 Éric de Chassey, « Paul Strand, Frontalité et engagement », Études photographiques, 13 juillet 2003, pp. 136-157. 
Loeb se donnant ainsi le temps de les faire réimprimer. Ces exemples illustrent les va-et-vient éditoriaux de Broom, qui doit composer avec les aléas de la production " délocalisée », qui n'assure pas toujours une impression aisée et une qualité de papier homogène. La provenance internationale des contributions, puisées aux États-Unis et en Europe (France, Italie, Allemagne pour la majorité), demande aux éditeurs une grande flexibilité, la collecte des articles et des illustrations, puis leur traduction ou leur adaptation si nécessaire, pouvant repousser leur publication de plusieurs mois.
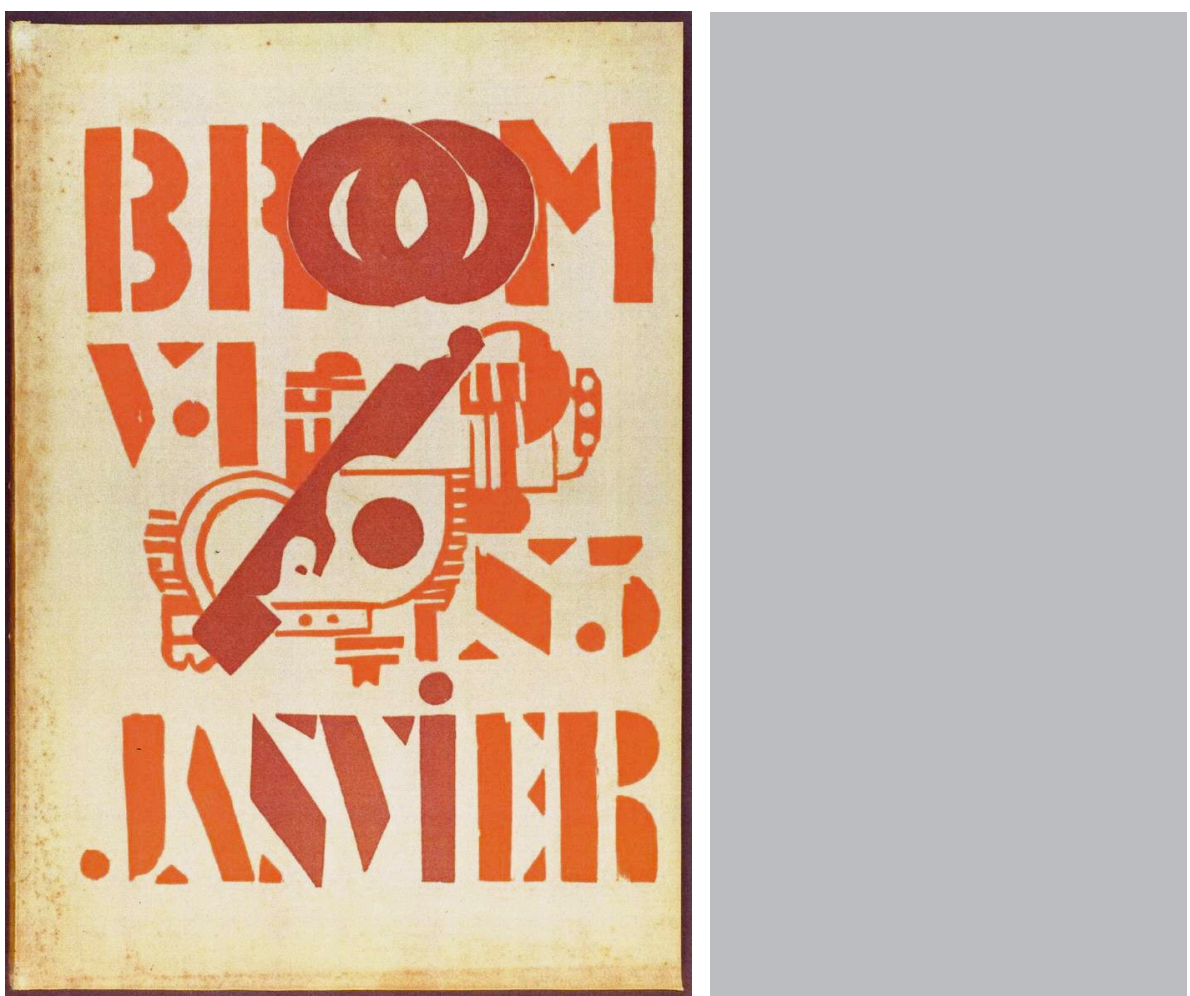


\section{Une ligne éditoriale internationale, entre modernisme américain et avant-gardes européennes}

S’intéressant à la littérature et aux arts plastiques internationaux, cette ligne éditoriale initiale est énoncée dès le premier numéro dans le «Manifesto I » (fig. 5) : la revue « sélectionne parmi la littérature continentale contemporaine des écrits de qualité exceptionnelle, les plus adaptés à la traduction en anglais ${ }^{24} \gg$. De la poésie expérimentale (E. E. Cummings, Wallace Gould, John Gould Fletcher, John Lee Masters) au renouvellement de la prose américaine (Sinclair Lewis, John Dos Passos, Jean Toomer), de la littérature contemporaine française (Pierre Reverdy, Louis Aragon, Paul Éluard, Philippe Soupault, Jean Cocteau) à ses sources fondamentales (Guillaume Apollinaire, Lautréamont), la revue tente de concilier des écrits novateurs en provenance des États-Unis et de l'Europe.

\section{MANIFESTOI.}

BROOM IS SFLECTING FROM THE CONTINENTAL, LITERATURE OF THE PRESENT

TTME, TTEE WRTTINGS OF EXCEPTTONAL, QUALTTY MOST ADAPTABLIF FOR TRANSI,ATION INTO ENGLISH.

THFSE WILI APPEAR SIDE BY SIDE WTTH THE CONTEMPORANEOUS EFFORT IN Great Britain and Amertca.

THIF PAINTERS AND SCUI,PTORS WILL BE REPRESENTED BY THE BEST AVAILABL,E RTPRODUCTIONS OF THEIR WORK.

THROUGHOUT, THE UNKNOWN, PATH-BREAKING ARTIST WILL HAVE, WHEN HIS MATERIAL, MERT'S IT, AT IIEAST AN EQUAL, CHANCE WITH THE ARTTST OF ACKNOWI.FDGED REPUTATION.

IN BRIEF, BROOM IS A SORT OF CLEARING HOUSE WHERE THE ARTISTS OF THE PRESENT TIME WTLI, BE BROUGHT INTO CLOSER CONTACT.

THE PERMANENCE OF THIS PROJFCT IS ASSURF:D ABSOLUTHLY IF SUPPOR'TED

BY THE SUBSCRIPTIONS OF THOSE SYMPATHETIC TO IT.

BECAUSF OF THE DISTANCE FROM 'THE SOURCE OF SUPPT, IT IS IMPOSSIBLE TO FILI THE RENEWAL ORDERS OF BOOKSHOPS AND TO CAI,CULATF THE QUANTTYY REQUTRED FROM AMERTCA.

YOUR SUBSCRTPTION (FIVE DOI,IARS A YEAR) MAILED AT THE EARLIEST POSSIBLFE MOMENT, SOLVES THIS PROBIFM FOR EVERYBODY CONCERNED.

AMTRICAN SUBSCRIPTIONS SHOULD BE MAITED TO THF NEW YORK OFFICE, THREE EAST NINTH STREIET; EUROPEAN SUBSCRIPTIONS TO THE MATN OFFTCE, I8, TRINITẢ DFI MONTI, ROMF; (6), ITALY

AND DO NOT FORGET TO SEND US THE NAAMES AND ADDRESSES OF YOUR FRIENDS

Les choix visionnaires de Loeb et Josephson permettent ainsi de faire découvrir Pierre Reverdy (1889-1960) au lectorat américain, alors même que l'écrivain peine à se faire connaître en France, que sa prose demeure réservée à des cercles d'initiés, affiliés au cubisme puis au surréalisme. Les textes sont publiés en anglais, ceci induisant un travail de traduction considérable pour toutes les contributions en provenance de l'Europe (France, Italie, Allemagne, Russie, Hongrie). Ces traductions sont pour la plupart réalisées par les éditeurs eux-mêmes (Kreymborg pour les textes italiens, Loeb et Josephson pour les textes français, Josephson pour les textes allemands). Elles accentuent ainsi la visée internationale de la revue, ceci permettant de tisser des liens ténus entre Amérique et Europe, liens instaurés par la production « délocalisée » et les voyages des éditeurs en Europe. Si Broom concentre ses efforts sur la littérature, elle n'en reste pas moins ouverte aux arts plastiques, « les peintres et sculpteurs

${ }^{23}$ [Harold A. Loeb et Alfred Kreymborg], « Manifesto I », Broom I, n 1, novembre 1921, troisième de couverture.

${ }^{24}$ Id., Ibid. : "Broom is selecting from the continental literature of the present time the writings of exceptional quality most adaptable for the translation into English". 
[étant] représentés par les meilleures reproductions disponibles de leur travail ${ }^{25} \%$. Ainsi, on retrouve dans ses pages des contributions d'artistes américains comme Joseph Stella, français comme Pablo Picasso, Henri Matisse et André Derain, allemands comme George Grosz. Cependant, et même si elle ouvre largement ses pages aux auteurs connus, la revue entend bien faire la promotion de jeunes talents, puisque dans Broom, "l'artiste inconnu, innovant, pourra avoir, quand sa contribution le méritera, la même chance que l'artiste reconnu ${ }^{26} »$. Cette ouverture d'esprit, ce désir de faire se côtoyer novices et confirmés, va progressivement faire la réputation de Broom, en donnant une chance aux artistes émergents de se faire connaittre tout en étant rémunérés. La revue répond ainsi à l'impératif d'abriter en ses pages « une sorte de maison de défrichage où les artistes du temps présent rentreront en étroite relation ${ }^{27} »$. Il s'agit selon Matthew Josephson, éditeur associé à partir de novembre 1922, de parvenir à « créer une sorte de pont entre la culture de deux continents en présentant des sélections significatives de l'art et de la littérature de l'Europe contemporaine et de l'Amérique ${ }^{28} »$. Cette dichotomie Amérique/Europe, modernisme/avant-gardisme, occupe donc une place centrale dans la revue, Broom cherchant avant tout à définir un nouvel art américain qui n'exclurait pas les influences européennes, si celles-ci parviennent à enrichir les racines américaines sans dériver vers une acculturation nocive. Ainsi, « ce qui fait l'originalité de Broom au sein des revues modernistes anglo-américaines de la période de l'entre-deux-guerres, c'est son ouverture au vaste espace culturel américain, par-delà le domaine des lettres et des arts visuels ; à travers les pages de la revue, nous voyons l'Amérique s'observer de près et de loin afin de se reconnaitre en tant qu'espace aux frontières fluides, recherchant ses spécificités, voire son altérité, face aux modernismes et aux avant-gardes de l'Europe ${ }^{29}$ ». Une double mission est donc assignée à Broom par ses éditeurs : la revue doit devenir un lieu ouvert de dialogue entre les États-Unis et l'Europe, tout en soutenant la thèse que certaines propositions artistiques ou politiques des avant-gardes européennes pourraient se greffer sur l'art américain pour l'enrichir. D'une part, elle tend à intégrer l'intention sociale et esthétique des avant-gardes européennes (à savoir, la fusion de l'art et de la vie) dans une perspective américaine, et de l'autre, à dissocier coûte que coûte l'expérience américaine de l'expérience européenne. Cette revue, publiée à 3266 miles de New York, 4269 miles de Chicago et à 6227 miles de San Francisco ${ }^{30}$, devient alors un exemple concret du rapport complexe entretenu par les intellectuels américains avec l'Europe et ses influences. Entre exil en Europe et rejet d'une culture européenne dominante, retour aux États-Unis et acceptation des créations européennes, la revue illustre brillamment les errances, l'évolution de la pensée et enfin l'apaisement d'une génération américaine en quête d'elle-même et d'une redéfinition de sa culture nationale. Ces questionnements existentiels, nationaux et créatifs se déploient en corolle, numéro après numéro, permettant d'entrevoir les querelles culturelles existant alors entre l'Amérique et l'Europe, et plus encore entre New York et Paris. Cette dialectique américano-européenne, influencée par les débats identitaires américains de la revue The Soil (1916-1917) publiée par Robert Coady, permet à Broom de complexifier sa ligne éditoriale et de maintenir un souffle polémique permanent, qui atteint son paroxysme avec l'arrivée de M. Josephson dans le comité éditorial à l'été 1922. Également influencés par la culture populaire présente dans les pages de The Soil, Loeb et Josephson intègrent dès lors dans leur revue le cinéma, esthétique mécanique de l'ère industrielle et le

\footnotetext{
25 [Harold A. Loeb et Alfred Kreymborg] op. cit. note 23 : "The painters and the sculptors will be represented by the best available reproductions of their work".

26 Id., Ibid. : "Throughout, the unknown, path-breaking artist will have, when his material merits it, at least an equal chance with the artist of acknowledged reputation".

27 Id., Ibid. : "Broom is a sort of clearing house where the artists of the present time will be brought into closer contact".

$28 \mathrm{M}$. Josephson, op. cit. note 4, p. 168 : "But the opportunities that opened for Broom were those of creating a sort of bridge between the culture of the two continents by presenting significant selections of the art and literature of contemporary Europe and America".

29 Stamatina Dimakopoulou, «Broom (1921-1924) : Avant-gardes, Modernités et I'Amérique retrouvée », Revues modernistes anglo-américaines : lieux d'échanges, lieux d'exil, sous la direction de Benoît Tadié, Paris, Entr'revues, 2006, p. 114.

30 Broom, n³, février 1922, page publicitaire vantant les tarifs avantageux des abonnements à la revue.
} 
médium photographique par le biais d'essais, d'illustrations ou de publicités sans cesse renouvelés. Pourtant, contrairement à The Soil, Broom n'est pas un one-man magazine. Ses publications se nourrissent de l'inspiration de chacun des éditeurs, composant ainsi une ligne éditoriale mouvante et en constante évolution. Le travail collectif des éditeurs, la notion de communauté de pensée et d'échanges sont les éléments déterminants de cette publication. S'opposant en ce point à la revue The Ennemy (publiée postérieurement en 1927) de Wyndham Lewis, Broom considère l'isolationnisme et la pensée unique comme réducteurs, préférant miser sur le dialogue et l'addition des compétences en tant que moteurs de la création. Cette dialectique complexe, entre attraction et répulsion des apports européens, se double d'une seconde dialectique, celle du dépassement de l'opposition entre culture de masse et culture élitiste. Si Harold Loeb rejette tout d'abord la culture populaire et ses productions (photographies, films), sa position évolue avec le temps, si bien qu'entre 1922 et 1923, sa principale préoccupation est de parvenir à définir un nouveau système de valeurs où art et pouvoir économique, élites et masses ne s'opposent plus. En quête d'un idéal dont la revue se fait le vecteur, il tend à imposer la possibilité d'un art se réappropriant le pouvoir économique de la culture de masses ${ }^{31}$. Ces contradictions et changements de points de vue, portés par les questionnements et expériences personnelles des éditeurs, nourrissent la revue du premier au dernier numéro, faisant apercevoir le cheminement intellectuel d'une jeunesse américaine sans certitudes absolues, dont les idéaux sont aussi mouvants que les territoires qu'elle explore.

\section{Une singularité éditoriale revendiquée dans le panorama des little reviews américaines}

Broom, par définition, semble donc s'inscrire pleinement dans le courant de publication des little reviews contemporaines, s'illustrant par sa charge contestataire et son opposition à la culture de son temps ou à certaines de ses tendances. Ces publications sont souvent utilisées ${ }^{32}$ pour attaquer les modes d'expression conventionnels, en incarnant une véritable rébellion éditoriale et humaine contre les doctrines du bon goût traditionnel. En cela, Broom fait entièrement corps avec cette volonté d'opposition, d'engagement et de défense du patrimoine culturel américain. Par sa volonté première de défense des auteurs américains face à la domination intellectuelle de l'Europe, la revue rejoint les priorités des revues The Dial et The Little Review (1919-1924). La volonté de dialogue entre lecteurs, contributeurs et éditeurs, est également une donnée partagée par Broom et The Little Review. Les deux publications conçoivent en effet leurs pages comme des lieux de dialogues et d'échanges, où chacun, qu'il soit contributeur ou lecteur, peut exprimer son point de vue. Les deux lignes éditoriales mettent en place une conversation ouverte entre écrivains et lecteurs, par l'intermédiaire de rubriques, chroniques ou de publications de lettres. Mais au-delà de ces points communs, Broom dispose de caractéristiques propres, d'une innovation formelle et intellectuelle qui la distinguent définitivement des publications contemporaines. L'idée généralement véhiculée voudrait que Broom réponde au critère principal du little magazine, en ce qu'il est une publication moderniste à faible tirage et coût de production ${ }^{33}$. Ce constat n'est valable que si l'on oublie le budget mensuel de la revue, de 800 à 1000 \$, mais surtout les importants crédits et donations de la famille Guggenheim qui ont nourri la revue jusqu'en février 1923. Les crédits alloués à Broom sont en fait bien plus importants que ceux alloués à la majeure partie des little reviens, lui permettant de résister plus de trois ans et de publier vingt-et-un numéros, en essayant de garder le plus grand format possible et de payer jusqu'au bout ses contributeurs. A titre de comparaison, ceux du Dial, de Secession ou de The Little

31 Michael North, "Transatlantic Transfer: Little Magazines and Euro-American Modernism", Modernist Magazines Conference, Leicester, De Montfort University, 12 juillet 2007, p. 13 : "The difficult task that Loeb tries to accomplish in Broom, then, is not just to reverse his earlier negative attitude toward American popular culture, but also to determine a system of value in which the economic power of that culture might be re-appropriated for art".

32 Comme le démontrent Frederick J. Hoffman, Charles Allen et Carolyn F. Ulrich dans The Little Magazine: A History and a Bibliography, Princeton, Princeton University Press, 1947, p. 4.

33 Dickran Tashjian, Skyscraper Primitives: Dada and the American Avant-Garde, 1910-1925, Middle-town/CT, Wesleyan University Press, 1975, p. 6. 
Reviens n'étaient pas rémunérés pour leurs contributions, sauf à titre exceptionnel. De même, ces trois revues employaient un papier de mauvaise qualité, ne pouvant rivaliser avec les couvertures en couleurs et les reproductions d'œuvres d'art de Broom sur papier Rag. Ces fonds importants, malgré leur diminution progressive, permettaient aux éditeurs de donner une apparence luxueuse à la revue, imposant sa voix par un standing éditorial élevé, qui ne fut pas égalé dans les années 1920. De même, la longévité de la revue la distingue de bon nombre de ses contemporaines. Avec ses vingt-et-un numéros publiés sur plus de trois ans, Broom distance de loin Secession ${ }^{34}$ (1922-1924) qui ne publie que huit numéros ou Gargoyle 35 (1921-1923) et ses six numéros. Une autre originalité de Broom concerne la publication " délocalisée » de la revue. La majorité des little revienss étaient alors publiée aux États-Unis (The Dial, The Smart Set ou encore Contact $3^{3}$ ), bien que quelques autres éditeurs choisissent de produire leurs revues en Europe. Faisant partie de ce groupe d'éditeurs exilés atypiques, Arthur Moss publie sa revue Gargoyle depuis Paris de 1921 à 1922. Gorham B. Munson, aidé par M. Josephson, fait le choix de produire sa revue Secession (1922-1924) depuis Vienne, puis Berlin. Enfin, au cours de l'année 1924, l'édition de The Transatlantic Review, est transférée à Paris par Ford Madox Ford.

Broom, au cours de son existence, s'est donc tracé un chemin singulier dans le courant de publication des little reviens. Au fil des numéros, la revue montre sa capacité à employer les codes impartis au genre des little reviews (revue littéraire, faible tirage, défense d'un art américain, organe de contestation) puis à les détourner pour aboutir à la création d'un objet éditorial hybride, ne correspondant plus à un genre défini et figé. Sa mobilité, sa multiplicité d'éditeurs et de collaborateurs, tout comme son image luxueuse fondée sur des couvertures imprimées en couleur et une richesse d'illustrations sans précédent, font de Broom une revue unique. Surtout restée dans les mémoires pour l'abondance de ses contributions françaises, Broom est bien souvent considérée uniquement, et à tort, comme une des nombreuses revues américaines ayant contribuées à l'essor de Dada à New York comme le sont à juste titre The Blindman de Marcel Duchamp, The Soil de R. Coady et 291 d'Alfred Stieglitz, publiées entre 1915 et $1921^{37}$. Si la présence de contributions du groupe Dada et de ses alentours sont indéniables (Louis Aragon, Philippe Soupault, Richard Huelsenbeck, Roger Vitrac), leurs publications au sein de la revue montre à quel point les propositions venues d'Europe constituaient des moyens et non des fins pour les éditeurs. Les créations issues de Dada sont présentées au lectorat américain afin d'être intégrées puis adaptées aux problématiques spécifiques de la culture américaine (ère industrielle, capitalisme, culture populaire). Il n'est ainsi pas question de copier aveuglément un schéma européen inadaptable aux États-Unis en raison des trop nombreuses différences culturelles, sociologiques,

${ }^{34}$ Secession, reprint, New York, Kraus, 1967. Secession est une revue américaine publiée de 1922 à 1924 par Gorham B. Munson, qui se pose dès son premier numéro en rivale de Broom. Développant sensiblement le même propos littéraire et artistique, publiant les mêmes contributeurs, elle se démarque par sa verve et sa dimension critique plus affirmée. Publiée depuis Vienne et Berlin, elle comporte 8 numéros marqués par la personnalité belliqueuse de Gorham B. Munson. Matthew Josephson, est d'abord ami avec l'éditeur, puis éditeur associé durant l'année 1922, avant de rejoindre Broom. Sa démission et son engagement avec une revue concurrente lui attirent les foudres de Munson et envenime la concurrence entre les deux revues. Une lettre de Josephson à Loeb, datée du 20 octobre 1923 et conservée à la Princeton Library, fait état d'une réunion entre les éditeurs/contributeurs de Broom et de Secession qui devait pacifier la situation, mais qui se soldera par une rupture totale de communication entre les deux revues. 35 Gargoyle est une revue américaine publiée par Arthur Moss de 1921 à 1923 à Paris. Éditeur de The Quills (1917-1921), Moss est le premier éditeur de little reviews à délocaliser ses publications. Gargoyle publie tout au long de ses 6 numéros des écrits contemporains et des reproductions d'art, tout comme Broom. Par ailleurs, on retrouve dans les deux revues le même coéditeur, Lasdilaw Medgyes. La revue est surtout connue pour avoir été la première à publier des écrits d'Ernest Hemingway, ce qui lui permet de se faire remarquer par Robert MacAlmon qui publiera The Sun Also Rises en 1925.

${ }^{36}$ Contact est publiée à New York par Robert MacAlmon et William Carlos Williams de 1920 à 1923. Revue d'art et de littérature, elle défend la poésie américaine et les arts visuels, tout en essayant, comme son titre l'indique, d'établir un contact entre différentes sources littéraires, différentes cultures et panoramas culturels. De nombreux contributeurs de Contact collaborent simultanément à Broom et à Secession.

37 D. Tashjian, A Boatload of Madmen, Surrealism and the American avant-garde 1920-1950, New York, Thames and Hudson, 1995, XVI. 
idéologiques qui séparent les deux continents. La thématique de l'opposition entre Dada et modernisme, Europe et États-Unis est certes une constante au sein de la revue, dont les prérogatives évoluent année après année, mais n'est pas développée avec autant d'insistance que dans les revues The New Republic (où Edmund Wilson dramatisait la question) ou 291. Les premiers numéros de Broom positionnaient plutôt la revue en tant qu'organe anti-Dada, comme en atteste l'essai « America Invades Europe ${ }^{38}$ » d'E. V. Sanders. Avec véhémence, l'auteur déplore l'attraction exercée par Paris et la France sur les intellectuels américains, alors même que Loeb y a séjourné et a profité pleinement de la capitale artistique pour trouver des contributeurs au cours de l'année 1921. Ce rejet primaire s'éteint progressivement avec l'arrivée dans le comité éditorial de Josephson. Dès lors, et cela jusqu'au retour de la publication à New York, les pages de la revue se font le chantre des artistes parisiens proches du cercle Dada et de Tristan Tzara.

Broom s'éteint progressivement au cours de l'année 1923, publiant son dernier numéro en janvier 1924. Les problèmes de financement et de trésorerie induisent une baisse de la qualité des contributions, un amenuisement du nombre de pages, une perte d'enthousiasme partagée à la fois par les éditeurs et par leur lectorat. L'agonie de la revue est lente mais sans appel, accentuée par des motions de censure éloignant tout espoir d'accalmie. Sa publication au cœur de New York l'expose au puritanisme et à l'étroitesse d'esprit des commissions de censure, ce puritanisme que Loeb avait cherché à fuir en quittant son territoire natal. Le 14 janvier 1924, alors que le numéro de janvier doit paraître, les éditeurs apprennent que sa diffusion a été suspendue par le comité de censure des services postaux (section 480) ${ }^{39}$. La publication du texte de Kenneth Burke, Prince Llian ${ }^{40}$, jugé trop érotique par la commission de censure, fait fermer la rédaction. À la suite de Broom, bien d'autres revues perpétuent l'exil éditorial, comme Transition, This Quarter ou Tambour. Sa constante recherche d'une redéfinition de la culture américaine, son rejet de l'Amérique traditionnelle et des valeurs de la ploutocratie, permet à la revue et à ses éditeurs de créer une Amérique utopique, n'existant que sur papier glacé. Cette Amérique idéale, où les chemins de fer côtoient les champs de blé d'une Italie millénaire, où les dollars de Wall Street fusionnent avec la plume humaniste des philosophes des Lumières, où les frasques dadaïstes s'accordent gaiement avec les états d'âme puritains, ne subsiste que dans l'imagination des éditeurs et des lecteurs de Broom. Parcourir aujourd'hui les pages de cette revue revient à essayer de faire revivre un peu de l'idéalisme et de l'engouement qui ont nourri les éditeurs de 1921 à 1924, en prenant conscience du caractère fragile et immanent d'une telle entreprise.

${ }^{38}$ Broom I, n 1 , novembre 1921, p. 89.

39 Malcolm Cowley, Exile's Return, A Literary Odissey of the 1920's, New York, Penguin Books, 1994, p. 195 : "For, on January 14, in the midst of the tumult aroused by the battle, another blow had fallen. Broom had been suppressed under section 480 of the Postal Laws, which prohibits the mailing of contraceptives and other obscene matters".

${ }^{40}$ Broom V, n 1 , janvier 1924, p. 12. 
Référence électronique

Ambre Gauthier, «BROOM : An International Magazine of the Arts (1921-1924): une revue d'avant-garde américaine. ", Cahiers de l'École du Louvre. Recherches en histoire de l'art, histoire des civilisations, archéologie, anthropologie et muséologie [en ligne] no 3, octobre 2013.

URL : http://www.ecoledulouvre.fr/revue/numero3octobre2013/Gauthier.pdf

\section{Creative \\ (․) $\odot$}

\section{L'auteur}

Après un diplôme de second cycle à l'École du Louvre et une licence de droit du patrimoine culturel à l'Université Panthéon-Assas, Ambre Gauthier est actuellement doctorante à l'Université Paris X-Nanterre.

Sous la direction de Rémi Labrusse, elle prépare une thèse sur les revues de galeries d'art en France dans l'entre-deux-guerres (1918-1940). Ses recherches portent sur l'histoire de l'art du XX $\mathrm{XX}^{\mathrm{e}}$ siècle, l'édition et le marché de l'art. Après avoir travaillé sur le catalogue de la collection de revues d'art Paul Destribats au Centre Pompidou, elle occupe le poste de chargée des expositions et des recherches à la Fondation Marc Chagall à Paris depuis 2011. 